\title{
Reproducibilidad de pruebas serológicas para el diagnóstico de infección por Trypanosoma cruzi en mujeres embarazadas de una zona endémica de Santander, Colombia
}

\author{
Yeny Zulay Castellanos ${ }^{1}$, Zulma Milena Cucunubá², Astrid Carolina Flórez², Luis Carlos Orozco-Vargas ${ }^{3}$ \\ 1 Facultad de Salud, Universidad Industrial de Santander, Bucaramanga, Colombia \\ 2 Grupo de Parasitología, Instituto Nacional de Salud, Bogotá, D.C., Colombia \\ ${ }^{3}$ Escuela de Enfermería, Universidad Industrial de Santander, Bucaramanga, Colombia
}

Introducción. El diagnóstico de infección por Trypanosoma cruzi en fase crónica se hace por medio de pruebas serológicas cuya reproducibilidad no está muy documentada.

Objetivo. Evaluar la reproducibilidad de las pruebas serológicas ELISA, IFI y HAI para el diagnóstico de infección por $T$. cruzi en mujeres embarazadas de una zona endémica de Santander.

Materiales y métodos. Mediante la evaluación de la tecnología diagnóstica se determinó la reproducibilidad de las pruebas serológicas ELISA, IFI y HAI en muestras de suero y elución sanguínea, seleccionadas mediante muestreo de corte transversal y pertenecientes a mujeres embarazadas de una zona endémica para enfermedad de Chagas en Santander. Se usó el software Stata, versión 10.0, para los análisis estadísticos. La prueba con la mejor reproducibilidad se determinó por medio de la comparación de los índices kappa más altos de cada técnica.

Resultados. Se evaluaron 777 sueros y elución sanguíneas. En suero, la prueba ELISA (punto de corte $=0,3$ ), la IFI (punto de corte $=1 / 32$ ) y la HAI (punto de corte=1/16) presentaron índices kappa mayores de $0,8\left(0,98, I C_{95 \%}: 0,93-1,00 ; 0,98, I C_{95 \%}: 0,92-1,00\right.$ y $0,88, I C_{95 \%}: 0,74-0,97$, respectivamente); no se observaron diferencias estadísticamente significativas entre las tres pruebas evaluadas $(p>0,05)$. Para la elución sanguínea, el índice kappa estuvo por debajo de 0,8 (valor kappa más alto: $0,55, I_{95 \%}$ : $0,41-0,68)$.

Conclusiones. Las tres pruebas serológicas presentaron reproducibilidad perfecta en suero, determinada mediante el índice kappa, por lo que cualquiera de ellas sería útil para establecer el diagnóstico de infección por $T$. cruzi. Por su simplicidad y su costo, la prueba ELISA se recomienda como prueba de elección para los programas de tamización de esta infección.

Palabras clave: Trypanosoma cruzi, enfermedad de Chagas, mujeres embarazadas, pruebas serológicas, reproducibilidad de resultados, salud pública.

doi: http://dx.doi.org/10.7705/biomedica.v34i2.1571

\section{Reproducibility of serological tests for the diagnosis of Trypanosoma cruzi infection in pregnant women in an endemic area of Santander, Colombia}

Introduction: The diagnosis of chronic Trypanosoma cruzi infection is supported by serological tests whose reproducibility has not been well documented.

Objective: To evaluate the reproducibility of the serological tests ELISA, IFAT and IHAT for the diagnosis of $T$. cruzi infection in pregnant women in an endemic zone in Santander.

Materials and methods: Through an evaluation study of diagnostic technologies, the reproducibility of the serological tests ELISA, IFAT and IHAT was determined in serum and eluted blood from pregnant women living in an endemic area for Chagas' disease in Santander. The samples were selected by cross sectional sampling. The software Stata ${ }^{\text {TM }}$ version 10.0 was used for statistical analysis. By means of the comparison of the highest kappa coefficient of each technique, the test with the best reproducibility was determined.

Results: A total of 777 samples were tested. In serum, ELISA (cutoff point: 0.3 ), IFAT (cutoff point: 1/32) and IHAT (cutoff point: 1/16) had kappa coefficients greater than $0.8(0.98,95 \% \mathrm{Cl}$ : 0.93-1.00; 0.98,

\footnotetext{
Contribución de los autores:

Yeny Zulay Castellanos: diseño del estudio, conducción del trabajo de campo, análisis de los datos, escritura del primer borrador del manuscrito

Zulma Milena Cucunubá: conducción del estudio, discusión de resultados, escritura del manuscrito

Astrid Carolina Flórez: elaboración de protocolos de procesamiento de muestras, coordinación de la realización de pruebas serológicas

Luis Carlos Orozco: diseño del estudio, análisis de los datos, elaboración del manuscrito

Todos los autores revisaron y aprobaron la versión final del manuscrito.
} 
95\% Cl: $0.92-1.00$ and $0.88,95 \% \mathrm{Cl}$ : 0.74-0.97, respectively); no statistically significant differences among the three tests were found $(p>0.05)$. For the blood eluates, kappa coefficients were below 0.8 (highest kappa: 0.55, 95\% Cl: 0.41-0.68).

Conclusions: For the three serological tests using serum, the reproducibility determined by the kappa coefficient was perfect. Selecting any of them is useful for the diagnosis of T. cruzi infection. Given its simplicity and cost, the ELISA test is recommended for screening for this infection.

Key words: Trypanosoma cruzi, Chagas disease, pregnant women, serologic tests, reproducibility of results, public health.

doi: http://dx.doi.org/10.7705/biomedica.v34i2.1571

La enfermedad de Chagas, causada por la infección por Trypanosoma cruzi, representa un problema de salud pública en Latinoamérica. En Colombia se estima que la población de zonas endémicas expuesta o en riesgo de infección es de 4'792.000 habitantes y el número de infectados es de 436.000 (1). Para el caso particular de las mujeres embarazadas, en Suramérica se han encontrado prevalencias que van de 1,7 a $80 \%(2-6)$.

El diagnóstico de la infección se hace mediante pruebas serológicas y parasitológicas, las cuales se emplean según la fase clínica en la que se encuentre el paciente. Así, en la fase crónica, cuando no hay parásitos en circulación, las pruebas serológicas son útiles dado que detectan la presencia de anticuerpos que indican un contacto previo con el parásito $(7,8)$.

La prueba ELISA (Enzime Linked Immunosorbent Assay) y la inmunofluorescencia indirecta (IFI) generalmente se emplean como pruebas para el diagnóstico de infección en la fase indeterminada y crónica de la infección (9); al usar la IFI como prueba de referencia, la sensibilidad de la ELISA puede estar por encima de $80 \%$ (10). Varios estudios han demostrado que, comparada con los resultados obtenidos por pruebas comerciales, la sensibilidad de las pruebas serológicas mejora si se usan cepas locales de $T$. cruzi como antígeno $(11,12)$.

Para las autoridades de salud pública de Colombia es importante identificar las mujeres embarazadas infectadas con $T$. cruzi, ya que de ello depende la vigilancia de la transmisión de la infección por la vía vertical (madre-hijo). Por lo tanto, se hace necesario establecer la calidad de las pruebas serológicas con las que se hace el diagnóstico de esta infección en zonas endémicas del país e

\footnotetext{
Correspondencia:

Yeny Zulay Castellanos, Carrera $16 \mathrm{~N}^{\circ} 7 \mathrm{~A}-53$, Cabecera II etapa, Piedecuesta, Santander.

Teléfonos: (577) 6542677 y (311) 5560208

yenyzu1@gmail.com

Recibido: 18/02/13; aceptado: 03/12/13
}

identificar la prueba que sea más apropiada para tal fin, aun más cuando el patrón de referencia para este evento no está claramente establecido.

El objetivo de este estudio fue determinar y comparar la reproducibilidad de las pruebas ELISA, IFI y de inhibición de hemaglutinación (Haemagglutination Inhibition, HAI), para el diagnóstico de infección por $T$. cruzi en mujeres embarazadas de una zona endémica para Chagas en el departamento de Santander.

\section{Materiales y métodos \\ Población, diseño y conducción del estudio}

En este estudio se evaluaron las tecnologías diagnósticas en fase II mediante un diseño de corte transversal en el cual se compararon tres pruebas serológicas en todas las muestras (13). Se identificaron todas las mujeres embarazadas que asistieron a control prenatal entre agosto de 2010 y septiembre de 2011 en 23 municipios de tres provincias (Guanentina, Comunera y García Rovira), que corresponden a una zona endémica para la enfermedad de Chagas en Santander.

Las mujeres embarazadas participaban en el programa piloto de vigilancia de enfermedad congénita de Chagas en Colombia (Cucunubá Z, Valencia C, Flórez C, León C, Castellanos Y, Cárdenas $\mathrm{A}$, et al. Pilot program for surveillance of congenital Chagas disease in Colombia, 20102011. $15^{\text {th }}$ International Congress on Infectious Disease. Bangkok, June 13-16, 2012. Int J Infect Dis. 2012;16(Suppl.1):53.011). Los criterios de inclusión fueron estado de embarazo, residencia en uno de los municipios seleccionados y aceptación de participar en el estudio mediante consentimiento informado.

\section{Cálculo del tamaño y selección de la muestra}

Se calculó el tamaño de la muestra teniendo en cuenta hallazgos previos con un coeficiente de correlación intraclase de 0,80 para la ELISA y de 0,76 para la IFI, y un poder de 0,9 (14), así como una proporción de diagnósticos positivos de 0,03 
y un nivel de confianza de $95 \%$, con base en lo cual se estimó incluir un mínimo de 700 muestras en el estudio. Teniendo en cuenta que en el banco de sueros se contaba con 1.518 muestras recolectadas de mujeres embarazadas en el periodo del estudio, se procedió a un muestreo aleatorio simple para la selección de una muestra representativa de la población.

\section{Pruebas de laboratorio}

La presencia de anticuerpos anti- $T$. cruzi se determinó mediante las pruebas ELISA, IFI y HAI. Para la prueba ELISA se evaluaron las absorbancias obtenidas en suero y en elución sanguínea a partir de muestras de sangre en papel de filtro; para IFI y HAl se evaluaron los títulos obtenidos en las dos lecturas realizadas con cada una de estas pruebas (figura 1).

El procesamiento de las muestras se llevó a cabo en el Laboratorio de Parasitología del Instituto Nacional de Salud por parte de dos bacteriólogas con más de cinco años de entrenamiento en diagnóstico serológico, quienes desconocían los resultados previos de las pruebas.

El antígeno empleado para las técnicas ELISA e IFI fue la cepa colombiana NV de T. cruzi previamente caracterizada como unidad discreta de tipificación (Discrete Unit of Typification, DTU) Tcl (15). Para la HAI se utilizó el estuche de la casa comercial Wiener, que utiliza glóbulos rojos de carnero sensibilizados con antígenos citoplásmicos de cepas de T. cruzi circulantes en el Cono Sur.
Los protocolos para el procesamiento de las pruebas fueron los estandarizados en el Laboratorio de Parasitología del Instituto Nacional de Salud. El montaje de las muestras para la ELISA se hizo por duplicado y su lectura fue la absorbancia emitida a $405 \mathrm{~nm}$ por el lector de placas de la institución. La IFI se hizo en diluciones desde 1:8 hasta 1:256 y el reporte del resultado fue la última dilución en la que se observó fluorescencia con objetivo de 40X en el microscopio de inmunofluorescencia. El procesamiento e interpretación de la HAI se hizo siguiendo las indicaciones del fabricante (16).

\section{Análisis estadísticos}

Los resultados de las pruebas se registraron en una base de datos en MS Exce ${ }^{\circledR}$ y se analizaron con Stata ${ }^{\circledR}$, versión 10.1 (17). Para conocer la concordancia entre las lecturas 1 y 2 de las pruebas ELISA en las muestras de suero y la lectura de la elución sanguínea, se utilizaron los límites de concordancia de Bland y Altman (13). Para evaluar la reproducibilidad de variables continuas, en este caso las absorbancias de la prueba ELISA, se empleó un coeficiente de correlación intraclase de 2,1 con un intervalo de confianza de $95 \%$. Cuando las variables eran dicotómicas se usó el índice kappa (18) medido en diferentes puntos de corte establecidos para cada prueba, así: para el ELISA, con absorbancias en densidad óptica de 0,3,0,4 y 0,5; para la IFI, con diluciones seriadas desde $1 / 32$ hasta $1 / 256$, y para la HAI, con diluciones seriadas desde 1/8 hasta 1/16.

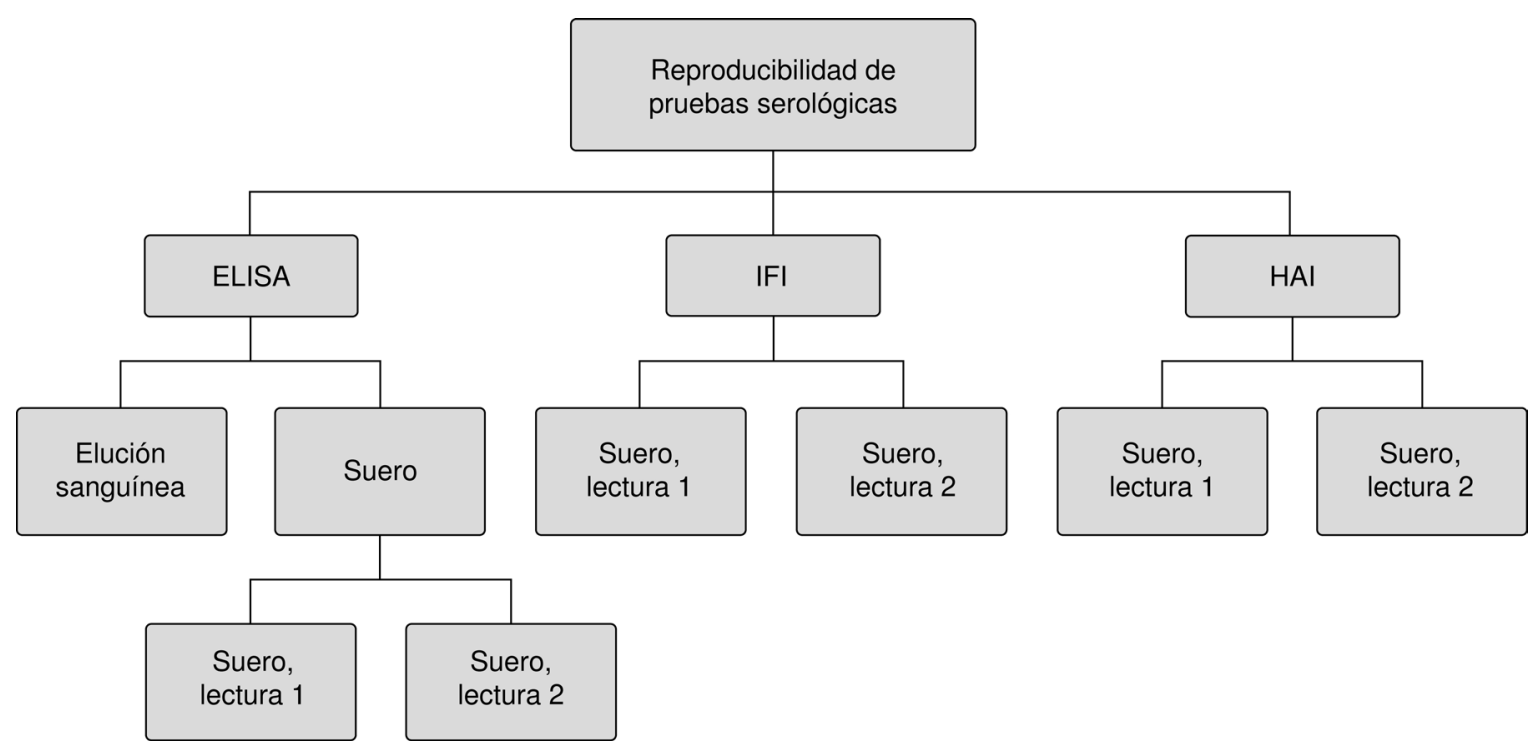

Figura 1. Flujograma del muestreo empleado para cada una de las pruebas serológicas evaluadas 
Para comparar la reproducibilidad de las tres pruebas, primero fue necesario seleccionar el mejor valor kappa obtenido para cada una de ellas. Dado que estos valores kappa correspondían a muestras dependientes, es decir pareadas, se usó la metodología desarrollada por Donner, et al., que requiere calcular varianza y covarianza (19). Una vez hecho esto, se calcularon y compararon los valores de $Z$ (20) y sus correspondientes valores de $p$.

\section{Consideraciones éticas}

El estudio se ajustó a los lineamientos de la Resolución No. 008430 del 4 de octubre de 1993 emitida por el Ministerio de Salud, por lo que se consideró una investigación de riesgo mínimo según el artículo II del capítulo I. La investigación fue aprobada por el Comité de Ética en Investigación del Instituto Nacional de Salud según acta 002 del 11 de julio de 2008, así como por el Comité ad hoc de Ética en Investigación de la Universidad Industrial de Santander bajo el código EPI 2033 del 15 de noviembre de 2012.

\section{Resultados}

\section{Caracterización demográfica de la población}

Se procesaron 777 sueros y eluciones sanguíneas a partir de muestras de sangre en papel de filtro pertenecientes a mujeres embarazadas de una zona endémica del departamento de Santander. Las participantes provenían de 23 municipios ubicados en las provincias Comunera $(37,8 \%)$, García Rovira (29,3\%) y Guanentá (32,8\%), y sus edades fluctuaban entre 13 y 45 años, con un promedio de 25 años $(\mathrm{DE}=6,89)$. Del total

2a. Suero, lecturas 1 y 2

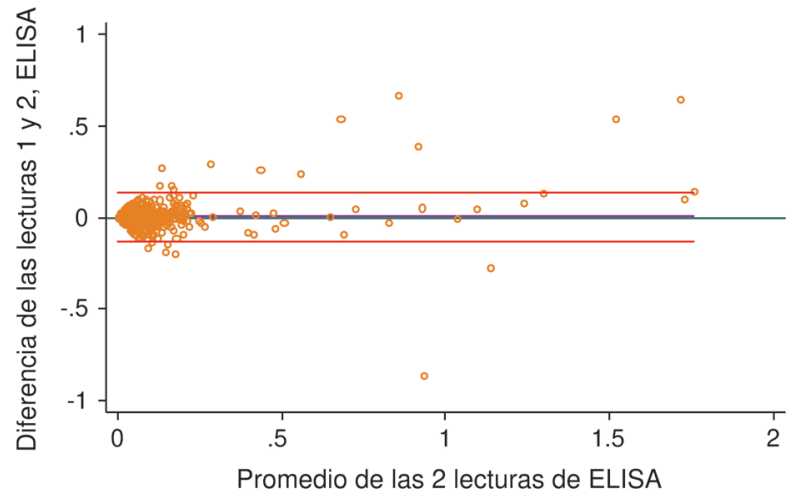

- Acuerdo promedio observado — Límites de acuerdo 95\% y=0 es la línea de acuerdo promedio perfecto

Figura 2. Límites de concordancia para la prueba ELISA
Cuadro 1. Caracterización de la población de mujeres embarazadas incluidas en el estudio

\begin{tabular}{|c|c|c|c|}
\hline Variable & $\mathbf{n}$ & $\%$ & $\mathrm{IC}_{95 \%}$ \\
\hline \multicolumn{4}{|l|}{ Provincia } \\
\hline Comunera & 294 & 37,84 & $34,42-41,35$ \\
\hline García Rovira & 228 & 29,34 & $26,16-32,68$ \\
\hline Guanentá & 255 & 32,82 & $29,52-36,24$ \\
\hline \multicolumn{4}{|l|}{ Procedencia } \\
\hline Área rural & 413 & 53,15 & $49,57-56,71$ \\
\hline Área urbana & 364 & 46,85 & $43,29-50,43$ \\
\hline Edad & $24,9^{*}$ & $6,89^{\star *}$ & $13-45^{\star \star *}$ \\
\hline \multicolumn{4}{|c|}{ Estrato socioeconómico } \\
\hline 1 & 534 & 68,90 & $65,33-71,97$ \\
\hline 2 & 216 & 27,87 & $24,67-31,09$ \\
\hline 3 & 22 & 2,84 & $1,78-4,26$ \\
\hline$>4$ & 5 & 0,4 & $0,21-1,49$ \\
\hline
\end{tabular}

${ }^{\star}$ : promedio; ${ }^{* *}$ : desviación estándar; ${ }^{* * *}$ : rango

de participantes, 68,9 \% pertenecía al estrato socioeconómico 1, 53,15\% procedía del área rural, y 46,85\% del área urbana (cuadro 1).

\section{Concordancia de la ELISA en suero y en papel de filtro}

Para determinar la concordancia entre las pruebas ELISA en muestras de elución sanguínea y de suero, se utilizaron los límites de concordancia de Bland y Altman. Las lecturas 1 y 2 en suero fluctuaron entre $-0,13$ y 0,14 , la desviación estándar fue de 0,07 y el promedio de la diferencia entre las mediciones fue de $-0,003$. Los valores de absorbancia estuvieron por debajo de 0,3. Cuando la absorbancia fue superior a 0,5 , los valores se salieron de los límites de concordancia (figura 2a). Para el caso de la comparación de las lecturas en suero con las de elución sanguínea,

2b. Elución sanguínea y suero

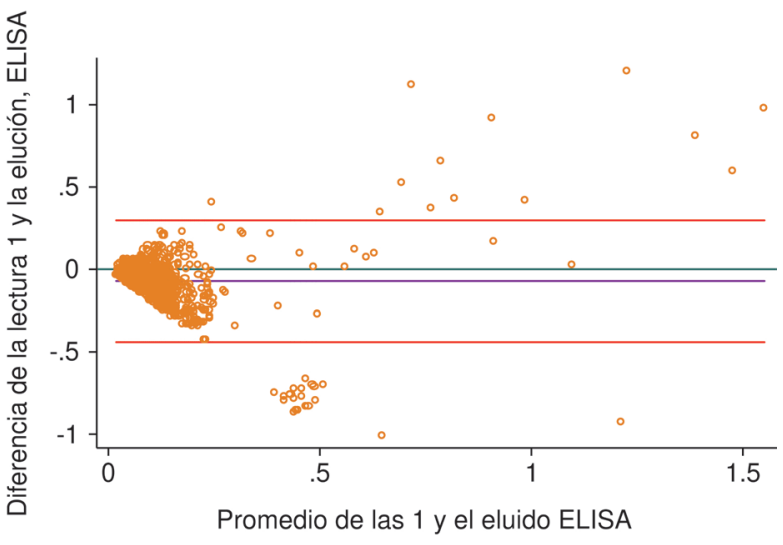

- Acuerdo promedio observado — Límites de acuerdo 95\% $\mathrm{y}=0$ es la línea de acuerdo promedio perfecto 
los límites de concordancia estuvieron entre - 0,29 y 0,45, la desviación estándar fue de 0,19 y el promedio de la diferencia entre las mediciones fue de 0,078. La comparación de la ELISA en suero con la de la elución sanguínea dejó ver que los valores de absorbancia se concentraron por debajo de 0,3. Con absorbancias mayores de 0,4 , los datos se dispersaron en igual proporción por encima y por debajo de 0,5 . No obstante, cuando la absorbancia se situó en alrededor de 0,5 apareció un grupo de datos por encima del límite superior (figura $2 b$ ).

\section{Reproducibilidad de ELISA, IFI y HAI}

Para evaluar la reproducibilidad de las pruebas serológicas con los valores de absorbancia de la ELISA y los títulos de la IFI y la HAI, se utilizó el coeficiente de correlación intraclase $(2,1)$. La mejor reproducibilidad se observó para las pruebas de ELISA en suero con un coeficiente de correlación intraclase de 0,92 (cuadro 2).

El comportamiento de los resultados de las pruebas ELISA, IFI y HAI como variables dicotómicas, se determinó mediante el cálculo del índice kappa con su respectivo intervalo de confianza de $95 \%$ para cada uno de los puntos de corte establecidos.
El valor kappa más alto para la IFI se registró en la dilución de 1/32: 0,98 $\left(\mathrm{IC}_{95 \%} 0,94-1,00\right)$. Para la HAI fue de $0,88\left(\mathrm{IC}_{95 \%}: 0,74-0,97\right)$ con punto de corte en cualquiera de las diluciones evaluadas. Para la ELISA en elución sanguínea y las lecturas 1 y 2 en suero, fue de $0,55\left(\mathrm{IC}_{95 \%}, 0,41-0,68\right)$ con un punto de corte de absorbancia de 0,4; para las lecturas 1 y 2 de la ELISA en muestras de suero fue de $0,98\left(\mathrm{IC}_{95 \%}: 0,94-1,00\right)$ con un punto de corte de 0,3 (cuadro 3).

Para la comparación de las tres pruebas serológicas se empleó el mejor índice kappa individual obtenido en cada una de las pruebas, así: para la ELISA en suero, en el punto de corte correspondiente a $0,3 \quad\left(0,98 ; \quad I_{95 \%} \quad 0,93-1,00\right)$, para la IFI, el correspondiente a una dilución de $1 / 32\left(0,98 ; I C_{95 \%} 0,92-1,00\right)$ y, para la HAI, en una dilución de $1 / 16\left(0,88 ; I_{95 \%} 0,74-0,97\right)$. Teniendo en cuenta la dependencia de las muestras, se utilizó la fórmula presentada por Donner, et al. (20). Los valores de $p$ calculados permitieron establecer que no hubo diferencias estadísticamente significativas entre las pruebas ELISA, IFI y HAI (cuadro 4).

La prevalencia de infección, entendida como un resultado positivo en las tres pruebas procesadas, cada una dos veces (es decir, seis pruebas en

Cuadro 2. Valores de coeficiente de correlación intraclase para la prueba ELISA en muestras de suero y elución

\begin{tabular}{lcc}
\hline Pruebas & CCI 2,1 $^{-1}$ & IC $_{\mathbf{9 5 \%}}$ \\
\hline ELISA en suero, lectura 1 Vs. ELISA en suero, lectura 2 Vs. ELISA en elución sanguínea & 0,58 & $0,49-0,65$ \\
ELISA en suero, lectura 1 Vs. ELISA en suero, lectura 2 & 0,92 & $0,91-0,93$ \\
ELISA en suero, lectura 1 Vs. ELISA en elución sanguínea & 0,43 & $0,31-0,53$ \\
ELISA en suero, lectura 2 Vs. ELISA en elución sanguínea & 0,40 & $0,26-0,50$ \\
\hline
\end{tabular}

CCl: coeficiente de correlación intraclase

Cuadro 3. Valores kappa para las tres pruebas practicadas y para los diferentes puntos de corte analizados

\begin{tabular}{lccc}
\hline Prueba & Punto de corte & Kappa & IC $_{95 \%}$ \\
\hline IFI, lectura 1 Vs. IFI, lectura 2 & $1 / 32$ & 0,98 & $0,92-1,00$ \\
& $1 / 64$ & 0,95 & $0,86-1,00$ \\
& $1 / 128$ & $0,84-0,95$ & $0,43-0,86$ \\
HAI, lectura 1 Vs. HAI, lectura 2 & $1 / 256$ & 0,69 & $0,74-0,97$ \\
ELISA en elución sanguínea Vs. ELISA en suero, lectura 1 & $1 / 8$ & 0,88 & $0,74-0,97$ \\
& 0,3 & 0,88 & $0,21-0,42$ \\
ELISA en elución sanguínea Vs. ELISA en suero, lectura 2 & 0,4 & 0,31 & $0,39-0,68$ \\
& 0,5 & 0,55 & $0,31-0,64$ \\
ELISA en suero, lectura 1 Vs. ELISA en suero, lectura 2 & 0,3 & 0,48 & $0,21-0,43$ \\
& 0,4 & 0,31 & $0,41-0,68$ \\
& 0,3 & 0,35 & $0,22-0,55$ \\
& 0,4 & 0,98 & $0,93-1,00$ \\
\end{tabular}


Cuadro 4. Comparación de los valores kappa de las pruebas serológicas evaluadas

\begin{tabular}{lccc}
\hline Dato & HAI Vs. ELISA & IFI Vs. HAI & ELISA Vs. IFI \\
\hline Covarianza & $-2,7 \times 10^{-4}$ & $-2,8 \times 10^{-4}$ & $1,1 \times 10^{-5}$ \\
Valor Z & 1,58 & 1,5 & 0,08 \\
p a dos colas & 0,11 & 0,13 & 0,94 \\
p a una cola & 0,06 & 0,06 & 0,47 \\
\hline
\end{tabular}

total), fue de $2,32 \%$ en las siguientes condiciones: para la ELISA en suero, con punto de corte mayor o igual a 0,3 , para la IFI, mayor o igual a 1/32 y, para la HAI, mayor o igual a $1 / 16$. No obstante, al considerar un resultado positivo en, al menos, una prueba, la prevalencia alcanzó 3,73 \%. Al observar los datos individuales para cada una de las técnicas, los resultados variaron en un rango entre 2,45 y $3,60 \%$ (cuadro 5).

\section{Discusión}

Tanto la validez como la confiabilidad son características de la calidad de las pruebas diagnósticas. En la mayoría de los estudios publicados se ha evaluado la primera de tales características, midiendo la sensibilidad y la especificidad. Las pocas publicaciones que reportan análisis de reproducibilidad presentan debilidades por el tipo de muestreo usado y por el tamaño insuficiente de la muestra (21). Por consiguiente, se puede decir que las pruebas serológicas usadas en la definición para probar una infección por $T$. cruzi han demostrado ser sensibles, específicas y prácticas para establecer el diagnóstico (10), pero no reproducibles.

La importancia de establecer si una mujer embarazada está infectada o no, radica en que implica un seguimiento exhaustivo tanto de la madre como del recién nacido y la toma de decisiones acertadas en su tratamiento; en la madre, permite reducir el impacto de las complicaciones crónicas $y$, eventualmente, romper el ciclo de infección vertical en futuros embarazos (22-24).
El índice kappa de Cohen, o $K_{0.5}$, es un coeficiente recomendado como medida de la eficiencia ponderada de una prueba (25). Para el caso de la HAl, el valor kappa ponderado fue de 0,88 y el coeficiente de correlación intraclase de 2,1 fue de 0,881. Ambos coeficientes demuestran que la técnica cuenta con un grado perfecto de reproducibilidad (18). La prueba de IFI, con un punto de corte en la dilución de 1/32, presentó un valor kappa de 0,979, lo que indica también una reproducibilidad perfecta.

La ELISA se evaluó tomando los límites de concordancia requeridos para observar el comportamiento de las dos mediciones, ya fuera en muestras de eluciones o en sueros. En cualquier caso, los datos se concentraron alrededor del valor medio cuando la absorbancia de las muestras no superaba una densidad óptica de 0,3.

En la ELISA en elución sanguínea, el límite de concordancia y el coeficiente kappa no mostraron una buena reproducibilidad. Resultados similares encontraron Zicker, et al., quienes reportaron una mayor reproducibilidad en muestras de suero que en eluciones sanguíneas para el diagnóstico de infección por $T$. cruzi en una zona endémica de Brasil. No obstante, no es claro de qué manera se determinó la reproducibilidad reportada (10).

Contrario a los hallazgos obtenidos en este estudio, Orozco, et al., establecieron un índice kappa de 0,96 para la ELISA en muestras de elución sanguínea y la IFI en muestras de suero de pacientes procedentes de una zona endémica de Boyacá, Colombia (14). La baja reproducibilidad encontrada en el presente estudio para la prueba ELISA en elución sugiere revisar todo el protocolo (desde los insumos para obtener la muestra hasta los cálculos para interpretar los resultados) (26), dada su potencial utilidad en estudios de seroprevalencia en zonas rurales $(27,28)$.

En el caso de las lecturas de ELISA en muestras de suero con un punto de corte a una densidad óptica de 0,3 , el valor kappa fue de 0,981 , lo que

Cuadro 5. Prevalencias encontradas para infección por T. cruzi tomando como parámetro cada una de las pruebas serológicas con el punto de corte establecido

\begin{tabular}{lcccc}
\hline Prueba & Punto de corte & Número de reactivos & Porcentaje de prevalencia & IC $_{95 \%}$ \\
\hline IFI en suero, lectura 1 & $1 / 32$ & 25 & 3,22 & $2,09-4,71$ \\
IFI en suero, lectura 2 & $1 / 32$ & 24 & 3,09 & $1,99-4,56$ \\
ELISA en suero, lectura 1 & 0,3 & 28 & 3,60 & $2,41-5,17$ \\
ELISA en suero, lectura 2 & 0,3 & 27 & 3,47 & $2,30-5,02$ \\
HAI en suero, lectura 1 & $1 / 16$ & 24 & 3,09 & $1,99-4,56$ \\
HAI en suero, lectura 2 & $1 / 16$ & 19 & 2,45 & $1,48-3,79$ \\
\hline
\end{tabular}


se interpreta como una reproducibilidad perfecta. Cuando se emplearon puntos de corte más altos $(0,4$ y 0,5$)$, la reproducibilidad de la prueba siguió siendo perfecta. Esta cualidad se vio confirmada cuando la ELISA se estudió como variable continua con el coeficiente de correlación intraclase de 2,1, obteniéndose un resultado de 0,924.

Se ha sugerido que una única prueba serológica bien seleccionada podría ser suficiente para diagnosticar la infección por T. cruzi (29). Aunque se han desarrollado diferentes fórmulas matemáticas para comparar los valores kappa cuando las muestras tienen dependencia (30-32), en este estudio se empleó la metodología propuesta por Donner, et al., la cual requiere calcular la varianza y la covarianza (19). Los resultados obtenidos mostraron una reproducibilidad perfecta para las tres pruebas evaluadas al emplear muestras de suero, sin registrar diferencias estadísticamente significativas entre una y otra prueba $(p \geq 0,05)$. Es posible que con un tamaño de muestra mayor se hubieran encontrado diferencias estadísticamente significativas entre la IFI o la ELISA, con respecto a la HAI.

Aunque los acuerdos internacionales sugieren emplear más de una prueba serológica para establecer el diagnóstico de infección por $T$. cruzi (33), los hallazgos del presente estudio respaldan la recomendación de emplear la ELISA como la principal prueba de elección para el diagnóstico de anticuerpos anti-T. cruzi. Asimismo, los estudios de Orozco, et al., y de López, et al., demostraron que la ELISA preparado con antígeno de una cepa local presentó mejores cualidades diagnósticas que la IFI, considerada por algunos autores como la prueba de referencia $(14,34)$. Por otro lado, la IFI, aunque también con excelente reproducibilidad, tiene desventajas como requerir un microscopio de fluorescencia y que su resultado está sujeto a la habilidad y experiencia de quien la interpreta $(14,35)$.

Entre las limitaciones de este estudio debe mencionarse que no se utilizó el mismo antígeno autóctono en las tres pruebas, ya que en la HAl se utilizó un antígeno obtenido de cultivos de cepas circulantes en el Cono Sur. Por otro lado, solo se hizo una lectura de la elución sanguínea, por lo que no fue posible conocer la reproducibilidad de la ELISA a partir de muestras de papel de filtro.

Entre las fortalezas del estudio pueden señalarse el tamaño de la muestra, que permitió obtener un poder suficiente para inferir los resultados alcanzados, y el hecho de que es el primer estudio sobre la reproducibilidad de índices kappa pareados empleando muestras dependientes. Las tres pruebas serológicas evaluadas en muestras de suero presentaron una reproducibilidad perfecta, establecida por medio de un coeficiente kappa ponderado, por lo que con cualquiera de ellas se podría establecer el diagnóstico de infección por $T$. cruzi. Por sus características, la ELISA no requiere un equipo sofisticado, permite el procesamiento simultáneo de varias muestras, requiere una cantidad mínima de antígeno y de muestra, y tiene bajo costo; todo ello permite recomendarla como prueba de elección para el diagnóstico de la infección en investigaciones futuras. Sin embargo, es conveniente contemplar también el estudio de una posible reacción cruzada con otros hemoparásitos circulantes en el país, como es el caso de Trypanosoma rangeli.

\section{Agradecimientos}

Los autores expresan sus agradecimientos a la Secretaría Departamental de Salud de Santander y a los hospitales de los 23 municipios participantes, por su apoyo logístico en la recolección de las muestras, y a Lyda Muñoz, por su aporte en el procesamiento de las pruebas de laboratorio.

\section{Conflicto de intereses}

Los autores declaran que no existe conflicto de intereses en esta investigación.

\section{Financiación}

Este estudio se desarrolló en el marco del proyecto "Desarrollo e implementación de un programa piloto de vigilancia de Chagas congénito en Colombia", financiado por el Departamento Administrativo Nacional de Ciencia y Tecnología de Colombia, Francisco José de Caldas (Colciencias), contrato número 1203-459-21581, y cofinanciado por el Instituto Nacional de Salud y la Pontificia Universidad Javeriana. Además, el proyecto contó con el apoyo logístico de la Secretaría de Salud de Santander.

\section{Referencias}

1. Organización Mundial de la Salud, Organización Panamericana de la Salud. Consulta técnica sobre información, educación y comunicación en enfermedad de Chagas congénita. Montevideo: OPS; 2007.

2. Cucunubá ZM, Flórez AC, Cárdenas A, Pavía P, Montilla $\mathbf{M}$, Aldana $\mathbf{R}$, et al. Prevalence and risk factors for Chagas disease in pregnant women in Casanare, Colombia. Am J Trop Med Hyg. 2012;87:837-42. http://dx.doi.org/10.4269/ ajtmh.2012.12-0086

3. Romero M, Postigo J, Schneider D, Chippaux JP, Santalla JA, Brutus L. Door-to-door screening as a strategy for the detection of congenital Chagas disease in rural Bolivia. Trop 
Med Int Health. 2011;16:562-9. http://dx.doi.org/10.1111/j. 1365-3156.2011.02746.x

4. Apt W, Zulantay I, Solari A, Ortiz S, Oddo D, Corral G, et al. Vertical transmission of Trypanosoma cruzi in the Province of Choapa, IV Region, Preliminary Report (20052008). Biol Res. 2010;43:269-74. http://dx.doi.org/10.4067/ S0716-97602010000300002

5. Russomando G. Transmisión congénita de la enfermedad de Chagas en el Paraguay. Mem Inst Investig Cienc Salud. 2009;7:55-64.

6. Bern C, Verástegui M, Gilman R, Lafuente C, Galdós G, Calderón M, et al. Congenital Trypanosoma cruzi transmission in Santa Cruz, Bolivia. Clin Infect Dis. 2009; 49:1667-74. http://dx.doi.org/10.1086/648070.

7. Anthony $\mathbf{R}$, Johnson C, Sousa $\mathbf{O}$. Use of micro-ELISA for quantitating antibody to Trypanosoma cruzi and Trypanosoma rangeli. Am J Trop Hyg. 1979;28:969-73.

8. Gomes Y, Lorena V, Luquetti A. Diagnosis of Chagas disease: What has been achieved? What remains to be done with regard to diagnosis and follow up studies? Mem Inst Oswaldo Cruz. 2009;104:115-21. http://dx.doi.org/10.1590/ S0074-02762009000900017

9. Figuêredo-Silva J, Kaneda $\mathbf{Y}$, Tachibana H, Furushima R, Tateno S, Correia-Lima FG. Epidemiological survey of Trypanosoma cruzi infection in north-eastern Brazil using different diagnostic methods. Rev Inst Med Trop. 1991;33:193-8. http://dx.doi.org/10.1590/S003646651991000300005

10. Zicker F, Smith PG, Luquetti AO, Oliveira OS. Mass screening for Trypanosoma cruzi infections using the immunofluorescence, ELISA and haemagglutination tests on serum samples and on blood eluates from filter- paper. Bull World Health Organ. 1990;68:465-71.

11. Gutiérrez R. Métodos convencionales de diagnóstico. En: Angulo VM. Curso Taller Internacional Control y Manejo de la Tripanosomiasis Americana. Bucaramanga: Universidad Industrial de Santander; 1999. p. 16-9.

12. Enciso C, Montilla M, Santacruz M, Nicholls RS, Rodríguez A, Mercado M, et al. Comparación de la prueba de inmunofluorescencia indirecta, un inmunoensayo enzimático y la prueba commercial Chagatek para la detección de anticuerpos anti-Trypanosoma cruzi. Biomédica. 2004; 24:104-8.

13. Orozco LC. Fases y muestreos, o de cómo tomar las personas de una población para hacer el estudio.En: Medición en salud, diagnóstico y evaluación de resultados. Un manual crítico más allá de lo básico. Bucaramanga: División de Publicaciones UIS; 2010. p. 63-103.

14. Orozco LC, Camargo D. El diagnóstico serológico de infección por T. cruzi. En: Angulo VM. Curso Taller Internacional Control y manejo de la tripanosomiasis americana. Bucaramanga: Universidad Industrial de Santander; 1999. p. 25-7.

15. Falla A, Herrera C, Fajardo A, Montilla M, Vallejo GA, Guhl F. Haplotype identification within Trypanosoma cruzi I in Colombian isolates from several reservoirs, vectors and humans. Acta Trop. 2009;110:15-21. http://dx.doi. org/10.1016/j.actatropica.2008.12.003
16. Wiener lab Group. Catálogo de pruebas. Fecha de consulta: 4 de enero de 2013. Disponible en: http://www.wiener-lab. com.ar/wiener/catalogo/archivos/6377_chagatest_hai_ sp.pdf

17. STATA Corporation. STATA. Texas: Inc., College Station; 2009.

18. Seigel D, Podgor M, Remaley A. Acceptable values of kappa for comparison of two groups. Am J Epidemiol. 1992;135:571-8

19. Donner A, Shoukri M, Klar N, Bartfay E. Testing the equality of two dependent kappa statistics. Stat Med. 2000;19:373-87. http://dx.doi.org/10.1002/(SICI)1097-0258 (20000215)19:3<373::AID-SIM337>3.0.CO;2-Y

20. Roldán JA, Luna JD. Comparison of weighted kappa coefficients of multiple binary diagnostic tests done on the same subjects. Stat Med. 2010;29:2149-65. http://dx.doi. org/10.1002/sim.3939.

21. Orozco L, Camargo D. Evaluación de tecnologías diagnósticas y tipos de muestreos. Biomédica. 1997;7:321-4.

22. Centers for Disease Control and Prevention (CDC). Congenital transmission of Chagas disease - Virginia, 2010. MMWR Morb Mortal Wkly Rep. 2012;61:477-9.

23. Bern C, Montgomery SP, Herwaldt B, Rassi A Jr, MarinNeto JA, Dantas RO, et al. Evaluation and treatment of Chagas disease in the United States: A systematic review. JAMA. 2007;298:2171-81. http://dx.doi.org/10.1001/jama. 298.18.2171

24. Sosa-Estani S, Colantonio L, Segura EL. Therapy of Chagas disease: Implications for levels of prevention. J Trop Med. 2012;19:1-10. http://dx.doi.org/10. 1155/2012/292138

25. Kraemer H, Periyakoil VS, Noda A. Kappa coefficients in medical research. Stat Med. 2002;2:2109-29. http://dx.doi. org/10.1002/sim. 1180

26. Evengard B, Linder E. Standardization of a filter-paper technique for blood sampling. Ann Trop Med Parasitol. 1988;82:295-303.

27. Vázquez $\mathbf{S}$, Fernández $\mathbf{R}$, Llorente $\mathbf{C}$. Utilidad de sangre almacenada en papel filtro para estudios serológicos por ELISA de inhibición. Rev Inst Med Trop. 1991;33:309-11.

28. Contreras M, Salinas P, Sandoval L, Solís F, Rojas A. Utilidad de la ELISA-IgG en muestras de sueros y eluidos de sangre en papel filtro en el inmunodiagnóstico de la enfermedad de Chagas. Bol Chil Parasitol. 1992;47:76-81.

29. Brenière SF, Carrasco R, Miguez H, Lemesre JL, Carlier Y. Comparisons of immunological tests for serodiagnosis of Chagas disease in Bolivian patients. Trop Geogr Med. 1985;37:231-8.

30. Williamson J, Manatunga A. Modeling kappa for measuring dependent categorical agreement data. Biostatistics. 2000;1:191-202. http://dx.doi.org/10.1093/ biostatistics/1.2.191

31. Vanbelle S, Albert A. A bootstrap method for comparing correlated kappa coefficients. J Stat Comput Simul. 2008:78;1009-15. http://dx.doi.org/10.1080/009496 50701410249

32. Barnhart H, Williamson J. Weighted least-squares approach for comparing correlated kappa. Biometrics. 2002;58:1012-9. 
33. Organización Mundial de la Salud. Enfermedad de Chagas: control y eliminación. $63^{\circ}$ Asamblea Mundial de la Salud A63/17. Geneva: WHO; 2010.

34. López M, Duque S, Orozco LC, Camargo D, Gualdrón L, Cáceres E, et al. Inmunodiagnóstico de la infección chagásica por ELISA. Biomédica. 1999;19:159-63.
35. Malan A, Avelar E, Litwin S, Hill H, Litwin C. Serological diagnosis of Trypanosoma cruzi: Evaluation of three enzyme immunoassays and an indirect inmunofluorescent assay. $J$ Med Microbiol. 2006;55:171-8. http://dx.doi.org/10.1099/jmm. 0.46149-0 